\title{
Bringing Treasures to the Surface: Iterative Design for the Library of Congress National Digital Library Program
}

\author{
Catherine Plaisant, Gary Marchionini, Tom Bruns, Anita Komlodi, Laura Campbell*
}

\author{
Human-Computer Interaction Laboratory \\ and Digital Library Research Group \\ University of Maryland, College Park MD 20742 \\ (301) 405-2768, plaisant@cs.umd.edu \\ http://www.cs.umd.edu/projects/hcil
}

\author{
*Library of Congress \\ National Digital Library Program \\ Washington DC 20540
}

\begin{abstract}
The Human-Computer Interaction Lab worked with a team for the Library of Congress (LC) to develop and test interface designs for LC's National Digital Library Program. Three iterations are described and illustrate the progression of the project toward a compact design that minimizes scrolling and jumping and anchors users in a screen space that tightly couples search and results. Issues and resolutions are discussed for each iteration and reflect the challenges of incomplete metadata, data visualization, and the rapidly changing web environment.
\end{abstract}

\section{KEYWORDS}

digital libraries, web design, browse, java, dynamic query, preview, design process, search.

\section{INTRODUCTION}

A team from the Human Computer Interaction Laboratory (HCIL) at the University of Maryland has been working with a team at the Library of Congress (LC) to develop and test interface designs for LC's National Digital Library Program (NDLP). The goals of the collaboration were to establish a user-centered design team for the NDLP, to create interface prototypes that serve a wide range of users, and to develop a variety of tools and widgets that LC may incorporate into future implementations. Beginning in the fall of 1995, HCIL team members met regularly with an LC team composed of librarians, managers, and technical staff to identify key interface challenges and brainstorm design approaches and features. As part of these efforts an extensive user needs assessment was conducted to determine characteristics of LC users and the types of information needs they bring to the Library. The needs assessment report presents a user typology with nine type/task components and identifies a series of interface challenges broken into content and user strategies categories [1]. During the January-August, 1996 period, a prototype interface evolved over several iterations. The evolution of this prototype is the subject of this design briefing. It is important to note that the prototype is not meant as a design that will itself be fully implemented but rather a means to explore different design problems and suggest design techniques and widgets to the LC implementation team.

HCIL has collaborated successfully with LC in the past, most recently on the LC ACCESS interface--a graphical, touch-panel design that is used in the Library's Main Reading Room [2]. The current project goal was to foster a user-centered design team similar to the ACCESS team. HCIL has focused considerable effort on interfaces that allow users to browse and search collections of information and has been developing interface techniques such as dynamic queries [3], visual information seeking [4] and query previews [5-6]. Dynamic query interfaces extend direct manipulation to search tasks by closely coupling search specification with the display of results. These interfaces feature a visual representation of a database, (typically with a scatterplot or starfield display), a visual representation of a query using a collection of widgets (e.g. sliders), and tight coupling between these two components. Users browse the database by interacting with the interface widgets. Each change produces a new query, the results of which are immediately and continuously shown in the display, supporting a progressive refinement of the search, continuous reformulation of goals, and visual scanning to identify results. Query previews deal with large distributed databases, and use previews of the data to maintain real time feedback and limit access to the network.

A design objective for the LC project was to apply dynamic query and query preview techniques to make browsing and searching in the LC NDLP easy and effective. As the project progressed, the goal was sharpened to achieve a compact design that minimizes scrolling and jumping and anchors users in a screen space that tightly couples search and result services. Three major iterations (each included one or more revisions) are described here. The general procedure in each iteration was to sketch de- 
signs on paper during team meetings or in subgroups, HCIL would refine the skteches and use drawing tools to formalize the designs for team discussion. Finally, "mockups" were prepared using Netscape. The reviews were conducted with groups of 10 to 15 LC staff members including active members of the design team and others who mainly reacted to the designs. For early prototypes of specific widgets or mechanisms, specialized tools were used (e.g., the Information Visualization Exploration Environment (IVEE at http://www.ivee.com) for an early starfield display prototype, and Macromedia Director for an animation mockup). However, our aim was to use Netscape as much as possible to ensure adoptability by LC's implementation team and facilitate remote reviews. Each iteration is described below and the design rationale that led to the subsequent revision is discussed.

\section{ITERATION 1: USERS' NEEDS DESIGN}

After discussing various metaphors and critical features, a first prototype was sketched on paper and then mocked up in Netscape. Functionality for word search, browsing, help, and new topics was included in the prototype but much of the thinking in this iteration was shaped by the results of the user needs assessment. Having just observed the complexity of searching the vast and varied collections of the physical library (some collections even require a staff interview before use) the design tried to direct categories of users into customized ways to search the collections. It focused on "help" by defining a virtual information desk (VID) where users were strongly encouraged to begin their visit (it is the first link). The intention of the VID design was to provide customized help by asking users to classify their roles and information needs. Roles included:

Tourist/surfer

Novice researcher

Casual researcher

Expert researcher

Teacher

Information needs included:

Specific item

General information on a topic

Comprehensive information on a topic

Another aspect that we felt was important was to clearly indicate that only a very small portion of the entire LC collection is now available in digital form, to avoid users being frustrated by the narrow focus of the current system. An LC building icon was proposed with the tip of the building color coded to indicate this fraction (Figure 1.)

The first search function listed on the screen used filters. The needs assessment had shown that place, time, format and people were the most common characteristics of users' needs; sets of filters were "mocked up" to allow users to specify the scope of their searches (Figure 2). Filters included: geographic region (map), chronological period, and format (i.e. text, manuscript, photo, sound, movies etc.) We did not include a name filter for two reasons. First, many of the objects are collections rather than authored items; furthermore, assigning attribution and/or who is depicted in a work remains a key metadata (i.e. data about data) challenge for digital libraries. Second, we wanted to avoid long, scrolling lists of names in this design. Word searching was also included for general queries.

\section{Issues}

As this iteration was discussed by the HCIL/LC team and revised, several implementation issues emerged. First, the team found it impossible to agree on written names for the user types and information needs and how to prompt users to understand what the expressions meant (e.g. Are "researchers" or "surfers" good categories and how do people identify themselves to categories.) More significantly, what to do with the user inputs was debated. Creating 15 different interfaces for the different combinations of user role and need seemed extreme and the subgroups working on specific versions were not making any progress. Another problem with this design is that it buried the browse and search functions under the user introductory services.

\section{Resolution}

The team decided to shift focus from user categories to tools for browsing and searching that would fit the needs of several categories at once. The 15 categories were collapsed into 5 more general user types and it was decided to provide specific introductory tours for each. Users would not be asked to identify themselves but would select a tour guide that matched the goal of their visit. To deal with the need to further assist certain categories of users a guided search was envisioned.

\section{ITERATION 2: FOUR SERVICES DESIGN}

The next version made the four main functions (browse, search, VID, and new arrivals/hot topics) more explicit by using the new HTML "tables" to visually separate the functions but compact them onto one screen to avoid scrolling. (see Figure 3). The first version of this design had one link for each box (e.g. the browse box had one link "browse" leading to a new page of browse options "by time", "by place" etc.). In later versions of this iteration items were moved up from lower levels of the hierarchy to the first page to minimize jumping (the browse box of Figure 3 includes a series of links).

In this design, the order of functions is revised as compared to Iteration 1. Browse and search actions are the first items: browsing was given prominence and a search box is available on the opening screen. Continuing our effort to deal with varied users, three types of search were included: quick (simple query), guided (a tutorial-like guided set of selections), and enhanced (Boolean queries with field limitations and other advanced features).

The Guided Search is available from the Virtual Informa- 
tion Desk section of the homepage (Figure 4). Users are led through the process of setting filters screen by screen. An explanation of the actions is available in the middle of the screen, and setting the criteria is closely coupled with an estimate of the results size shown on the color coded result preview bar on the right. For example, when selecting a time period users can read that most materials are historical or out-of-copyright; they can see the distribution of volume of materials over the decades; and the bar on the right reflects the impact of their selection on the search scope, helping users learn about the content of the collection.

Results are presented in a framed list but it was envisionned that results could also be seen in a starfield display using metadata about the items such as date, size, type etc.

\section{Issues}

This design provided quick access to the tools but major concerns were now raised about feasibility. The lack of descriptive data (metadata) underlying different objects had become a central problem as a result of working with the two previous iterations. In the American Memory collections some objects are entire collections, some are finding aids that help users locate items in groups of items (e.g. a page in a manuscript collection), and some objects are individual items which ,in most cases, have no descriptive data. Those distinctions about object type are not always possible with a "search engine". Furthermore, most objects are not specific to a geographic region or time period or such metadata is not available in the underlying records, and many individual items are not catalogued at all. These challenges limited the usefulness of the general filters that could apply across all of the LC NDLP and make it difficult to construct adequate previews (e.g. the bars of figure 4). This led to the conclusion that additional metadata was necessary to design a good interface.

An additional concern was that the design focus on tools was still not bringing to the surface the treasures of the collections. Users still would have to navigate through several levels of the site hierarchy before being able to even see a sample of a collection and appreciate the value and coverage of the materials. Similarly, the rich "special presentations" written by domain experts about the materials were burried despite their great usefulness.

\section{Resolution:}

Samples of objects had to be brought forward, which implied a redistribution of the space for the tools on the front page.

Adding metadata is a necessity for comprehensive user access to individual items but doing so is prohibitively expensive for all items. A decision was made to add descriptive data only about collections. This was in harmony with the users' need assessment which showed that identi- fying the best collections for searching was critical. The guided tour had to be abandoned because of lack of data and the visualization effort moved to the collection level.

To summarize, this iteration accomplished the goals of (1) decreasing the need for scrolling and (2) flattening the navigation hierarchy; however data visualization was limited by the lack of underlying metadata and user support remained an issue.

\section{ITERATION 3: LEFT COLUMN TAB DESIGN}

Whereas the first iteration focused on introducing users to the LC NDLP and the second focused on the tools for browsing and searching, the third iteration focused on bringing forward the materials of the LC NDLP. We aimed to aid users by bringing them quickly and visually to interesting materials that would serve as a springboard for defining their needs. The coupling of search and results was made tighter by anchoring users in a single dynamic screen rather than forcing them to jump to new screens.

\section{Compact design}

The main functions were arranged in "tabs" down the left side of the screen. Results for currently selected functions were displayed on the remainder of the screen (using HTML frames). The "tabs" are always present on the left side of the screen, and since real tabs could not be implemented in HTML color coding was use to match the tab and the right frame. This design (see Figure 5) is more compact than previous iterations and goes further in coupling search and results. The persistence of the titles and tabs gives the impression that it is possible to browse lists of collections, specify complex searches, and review results without ever leaving the first page.

\section{Samples}

On the introduction page an animated series of samples from collections and links to the special presentations written by experts aid users in familiarizing themselves with the digitized materials. The accent in this iteration was on providing immediate access to some of the treasures of the collections instead of users' categories or impersonal search tools. Users often have a vague idea of what they hope to find and browsing samples help them shape their query.

\section{Collection browsing}

Since the decision was made by LC to ensure that adequate metadata woul be available at the collections level, a major step was to use Java to implement a dynamic query application for browsing and selecting collections. The tab below Introduction is "Browse and Select Collections" (Figure 6) which update the right frame to a graphical overview of all collections, a few filters, and a list of collections which can be sorted on a variety of attributes, such as date, topic, and formats. Technically, this implied a visual representation of the available Collections, and widgets that allowed dynamic filtering of Collections on 
various attributes. At the time this work began, web pages were generally static. Technologies such as server-push or CGI did not support the immediate response required for dynamic queries. JavaScript and dynamic GIFs were not yet available. The only client-side technologies that were sufficiently mature and available were Java and Shockwave/Director. HCIL chose Java because we had already started to work on a similar dynamic query interface for our NASA's EOSDIS project [5].

\section{Graphic overview}

Previously, starfield displays had been used to display "point" data, where database objects assume discrete values on each axis of the scatterplot. But few of the collection attributes could be represented as a single discrete value. Since the starting and ending dates defined an interval the starfield became an "interval" field, where each collection was represented by a horizontal bar, defining the $\mathrm{x}$-axis as "time." The y-axis posed a problem. What other attribute could the intervals be plotted against? Size of the collection was considered but the LC team was reluctant to define a size for each collection (is size the number of items, byte size, time to read or review?). In the end it was decided that the y-axis would represent no other attribute. Instead, the intervals would simply be iterated in a non-overlapping manner down the vertical axis of the interval-field. Color-coding schemes for the intervals were also considered, and rejected. Most of the attributes for the collections can assume multiple values. For example, the Format attribute can be any combination of Text, Film, Sound, etc., so a single color code would not be appropriate. The interval field can be panned and zoomed by manipulating the double-box slider beneath the field, also implemented in Java.

\section{Java Implementation}

The Java prototype has three main components: the Collection Overview, the Collection Filters, and the Collection List. The use of Java allowed the Collection Overview and Collection List to dynamically change in response to collection filters, showing only those that satisfy the filter constraints. Furthermore, the Collection Overview itself acts as a filter. Panning or zooming on a particular interval of time specifies a temporal constraint, potentially filtering out more collections. The three interface components are linked via an active cursor. Passing the cursor over a collection interval or collection name highlights the other, as well as the appropriate attributes in the Collection Filters. Pressing the mouse button causes a jump to the collection homepage. The collection list can be sorted on any of the collection attributes. The use of Java allows interface objects to be created dynamically, based on the contents of a text file that defines the collections. Java facilitates the active linking between components, links to homepages, and collection sorting. Most importantly, Java facilitates dynamic queries by allowing each discrete user event, such as a mouse moving a slider, to be intercepted and processed, triggering a re-draw of the visualization of results. This tight coupling of user events to graphical re-drawing is fundamental to dynamic queries.

\section{Search and results}

Quick Search is available from the first page and is meant to be run on all the collections or limited to the collections selected in the dynamic query interface presented above. Enhanced Search allows Boolean queries and gives access to the attributes common to the collections selected (e.g. restricting the search to photograph collections would add a new field to the Enhanced Search, allowing users to restrict the search to black and white or color photographs). Results are presented in tables which can be reordered according to any attribute. Glyphs could also be added to give information about type and approximate size of result items (e.g. a collection is distinguished from a photograph).

\section{Issues}

Feedback from the group shifted to implementation issues (how reliable is Java?) and graphical design (how to make those design prototypes more attractive?) which pushed us to sketch more polished graphic designs. As the project evolved it became clear that what was needed was not a self contained complete interface but a set of consistent interfaces for distinct collections and design directions for future interfaces. The team was encouraged to replace the planned usability testing with reviews by large audiences at LC and to explore the applicability of the design at other levels of the LC NDLP. In particular it was decided to apply the prototype design to the Coolidge Consumerism collection.

Design issues are now focusing on browsing the results list. Result lists are composed of heterogeneous materials (collections, items, finding aids, etc.) and the design needs to represent the different level of representation and provide overviews of the volume of material returned. Finally, once the results have been ordered and presorted it will remain a challenge for users to review large quantities of materials. For example if a search in photo collections returns 100 pictures, tools for rapidly browsing and using the most relevant images are needed.

\section{CURRENT DIRECTIONS: MORE BROWSING TOOLS}

As we continue our design process we are concentrating on tools to allow users to quickly scan scores of images and select some for more careful viewing or saving. For example a user preparing a photo report on Theodore Roosevelt needs to eliminate the many pictures of boats or bridges or family members, then flag the preferred views, and finally save them with annotations in a new page. See Figure 7 for an early prototype that allows users to quickly scan a set of thumbnails, examine full images and descriptive information, and flag those of interest for saving. Other tools are been built for other media (such as video or manuscripts) in order to provide fast access to preview materials, thus bringing the collection treasures to the 
front. Results can be saved in an automatically generated page which will assist users with limited time and computer knowledge (e.g. teachers) to immediatly use the NDLP items in their own materials.

\section{CONCLUSION}

This project illustrates iterative design for a large-scale digital library that will serve the needs of a wide range of users. The iterations demonstrate ways to progressively more closely link query and results (the essence of dynamic queries), collapse hierarchy, compact design, and minimize scrolling and jumping. The project also provides an experience base for designers working on the problem of mapping metadata to browse/search and visualization widgets. Of course we are still far from a satisfying interface ready for extensive user testing and answering all the difficult digital library problems. Key challenges include: finding ways to show different levels of representation in results; providing search services for objects that have inconsistent or no descriptive data; developing guided tours and support through the VID; and integrating these designs with the larger LC site.

\section{ACKNOWLEDGMENTS}

This work is supported in part by the Library of Congress. Laura Campbell is the Director of the National Digital Library Program. In addition to the authors, the LC NDLP Team Members were: Martha Anderson, Maryle Ashley, Ardie Bausenbach, Mike Black, Jeff Bridgers, Beth Davis-Brown, Ernie Emrich, Floris Flam, Georgia Higley, Lois Korzendorfer, Sandy Lawson, Betsy Mangan, Gary Fitzpatrick, Barbara Natanson, Virginia
Sorkin, Susan Veccia, Peter Yeager and Walter Zvonchenko. We also want to thank Ara Shirinian for his work on the image browsing tool and Teresa Cronnell for her participation.

\section{REFERENCES}

1. Marchionini, G., Plaisant, C., Komlodi, A., User needs assessment for the Library of Congress National Digital Library, Technical report CS-TR-3640 (also available from our project web pages at http://www.cs.umd.edu/projects/ hcil/Research/1995/ndl.html.)

2. Marchionini, G., Ashley, M., Korzendorfer, L. (1993) ACCESS at the Library of Congress,Sparks of Innovation in Human-Computer Interaction, B. Shneiderman, Ed., Ablex Publ., Norwood, NJ (1993) 251-258.

3. Ahlberg, C., Williamson, C., \& Shneiderman, B. Dynamic queries for information exploration: An implementation and evaluation. ACM CHI '92 Proceedings (Monterey, CA, May 3-7, 1992), 619-626.

4. Ahlberg, C., Shneiderman, B., Visual Information Seeking: tight coupling of dynamic query filters with starfield display, ACM CHI'94 Proceedings (Boston, MA, April 1994), 313317.

5. Doan, K., Plaisant, C., Shneiderman, B., Query previews in networked information systems, Proc. of the Third Forum on Research and Technology Advances in Digital Libraries, ADL '96 (Washington, DC, May 13-15, 1996) IEEE CS Press, 120-129. (Also a CHI 97 Video)

6. North, C., Shneiderman, B., Plaisant, C. User Controlled Overviews of an Image Library: A Case Study of the Visible Human, Proc. of the 1st ACM International Conference on Digital Libraries (Bethesda, MD, March 20-23, 1996) 74-82

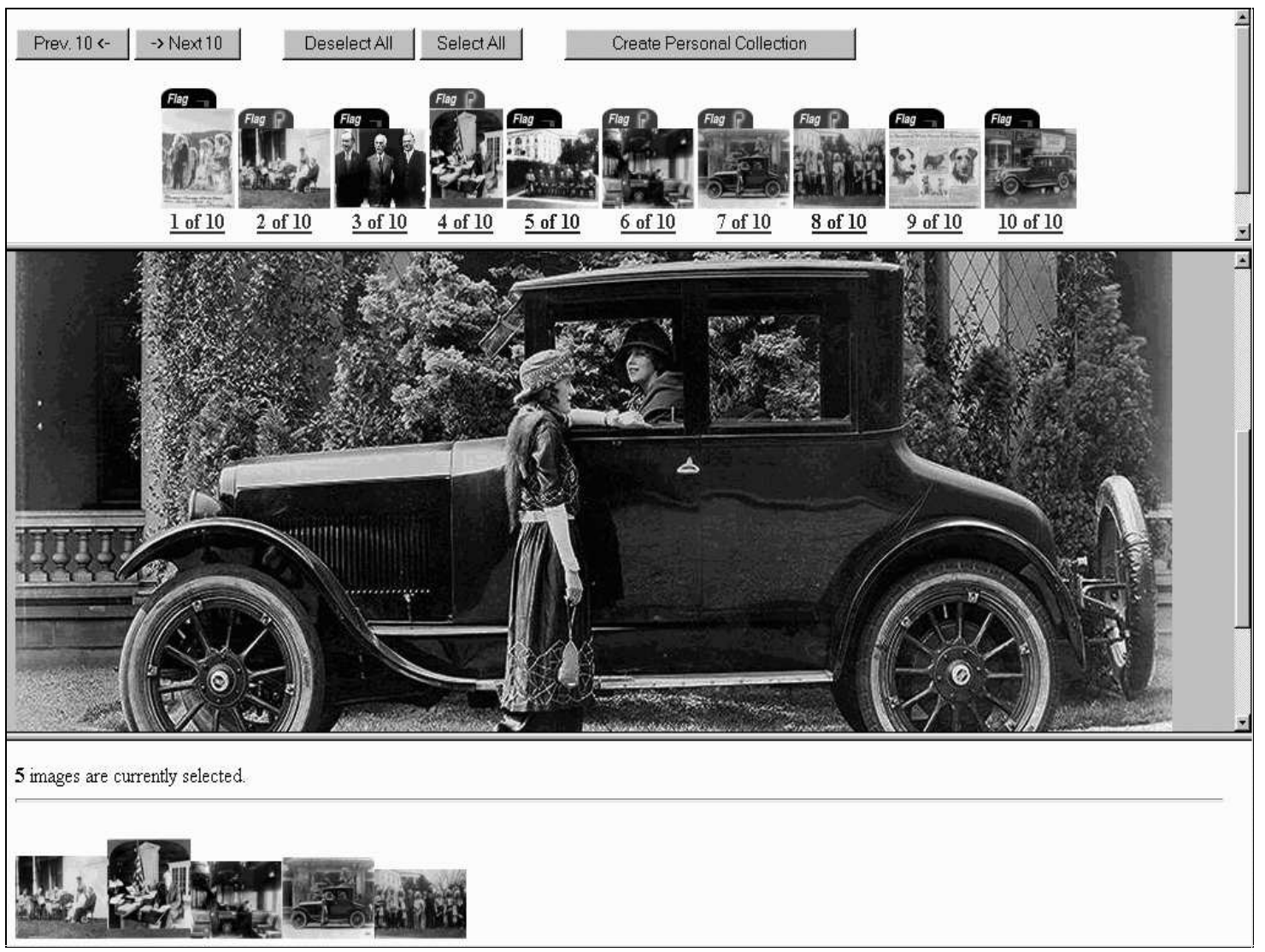

Figure 7:

One of the new tools developed to support image browsing. Here users can look at thumbnails and large images, select images and create their own collections of images. 


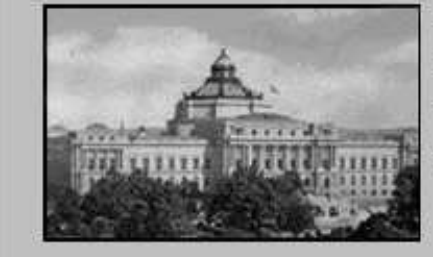

\section{Library of Congress - National Digital Library Program}

\section{WELCOME !}

What is in the Digital Collections?

A small portion (represented by blue in the figure on the right) of the huge collections of the Library of Congress is digitized. The Digital Collections mainly include histoncal and uncopyrighted or out-of-copyright materials. It is a constantly growing collection that contains both primary materials and secondary materials (e.g. indexes, finding aids).

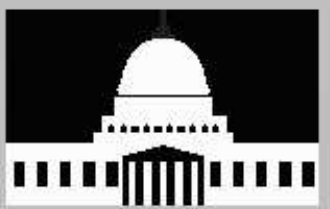

To make your visit worthwhile, first visit the Virtual Information Desk.

- Virtual Information Desk

O version one

0 version two

- Hot Topics

- New Anvials

Specify your action

- Set filters (chronological scope, geographical area format)

- Chose topic

- Choose name

- Specify query
Figure 1: The home page in Iteraction 1 gives a short description of the collection and a graphic in the right to illustrate the proportion of the digitized materials to the whole collection at the Library of Congress. This screen uses a menu layout to present the options starting with user support services (Virtual Information Desk, Hot Topics, New Arrivals), and providing search and browse actions in the second part of the menu.

\section{Set your filters:}

\section{Geographical area:}

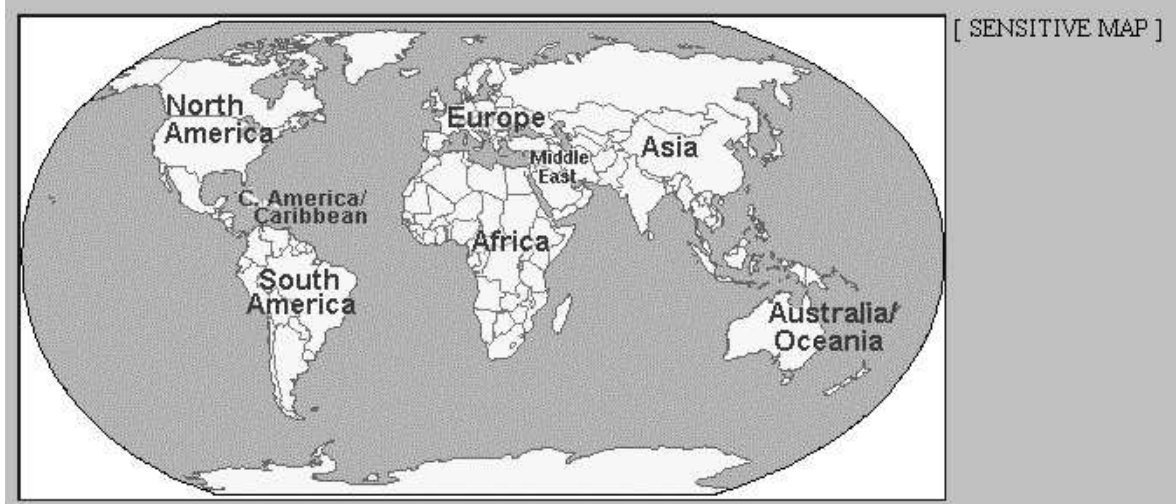

Chronological scope:

\begin{tabular}{|l|l|l|l|l|l|l|l|l|l|l|l|}
\hline \multicolumn{5}{|c|}{1700} & \multicolumn{5}{c|}{1800} & \multicolumn{4}{c|}{1900} \\
\hline 25 & 50 & 75 & 99 & 25 & 50 & 75 & 99 & 25 & 50 & 75 & 99 \\
\hline
\end{tabular}

Format:
c Text.
$c$ Image
$c$ Sound.
C Map.
C Motion Picture.

Figure 2: This figure shows a part of the "Set Filters" screen from Iteration 1. It provides customized graphical filters to the attributes found most important to users. After setting each filter a color coded result bar shows the amount of materials relevant to the attribute ranges selected. 


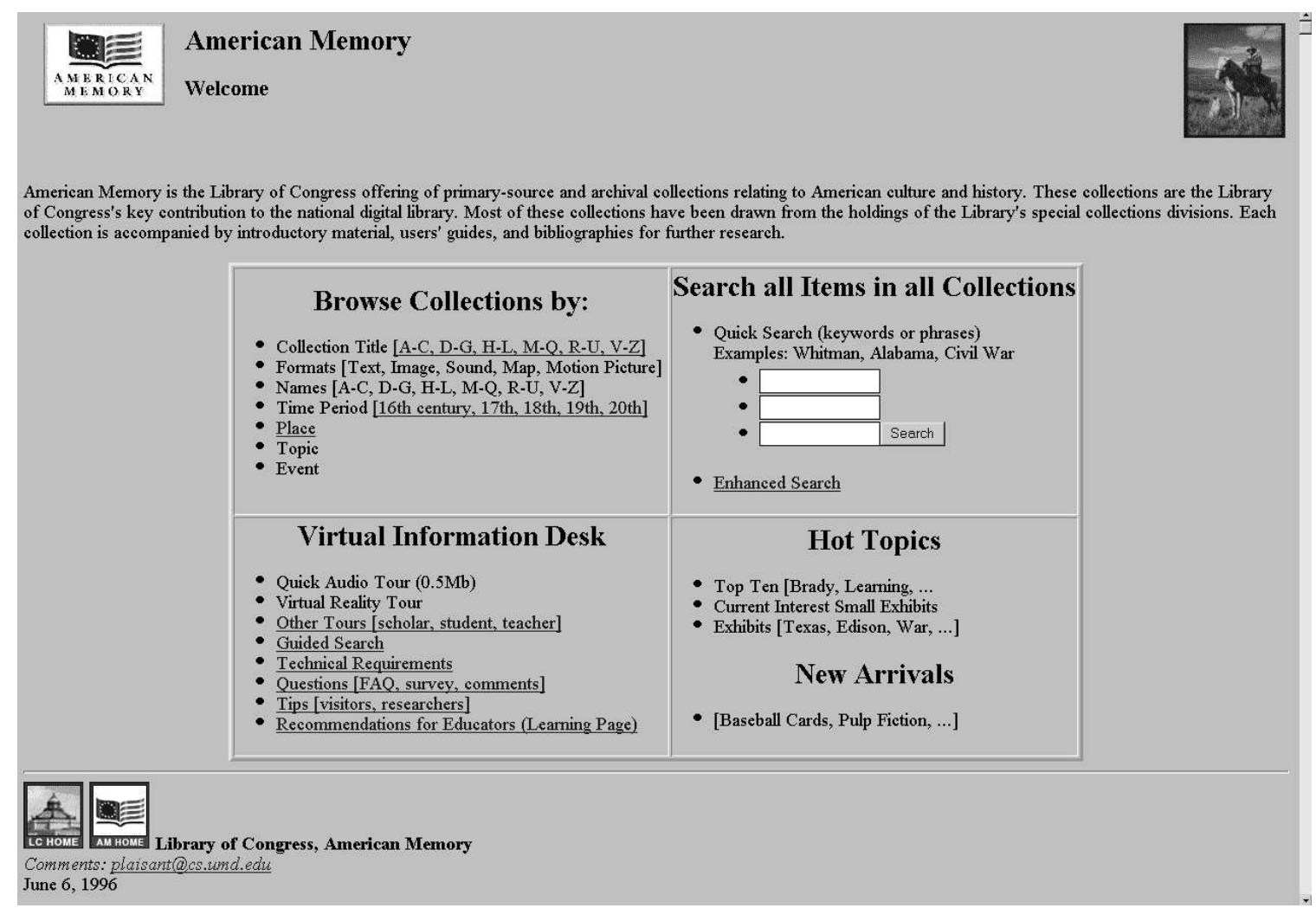

Figure 3: The homepage in Iteration 2 is compacted into a four-box design saving space. This page moves items up from lower levels of the hierarchy to the first page to minimize jumping. Here the order of services is revised as compared to Iteration 1, browse and search actions are the first items, and a search box is available right on the opening screen.

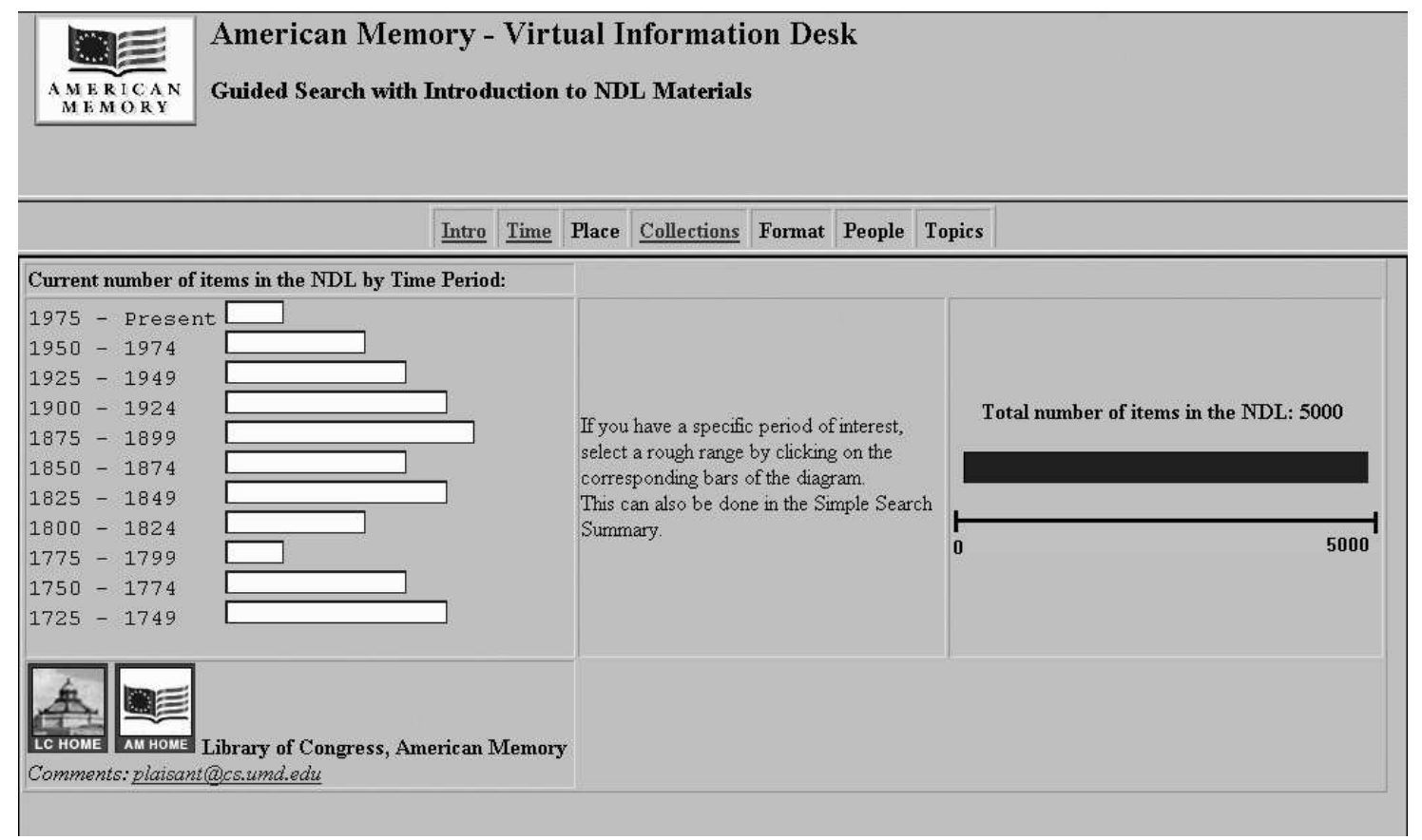

Figure 4: Guided search is available in Iteration 2 from the Virtual Information Desk section of the homepage. Users are led through the process of setting the filters screen by screen. An explanation of the actions is always available in the center, while setting the criteria is closely coupled with results shown on the color coded bar on the right. The bars on the left indicate the amount of materials relevant to a period and help users learn about the content of the collection. 


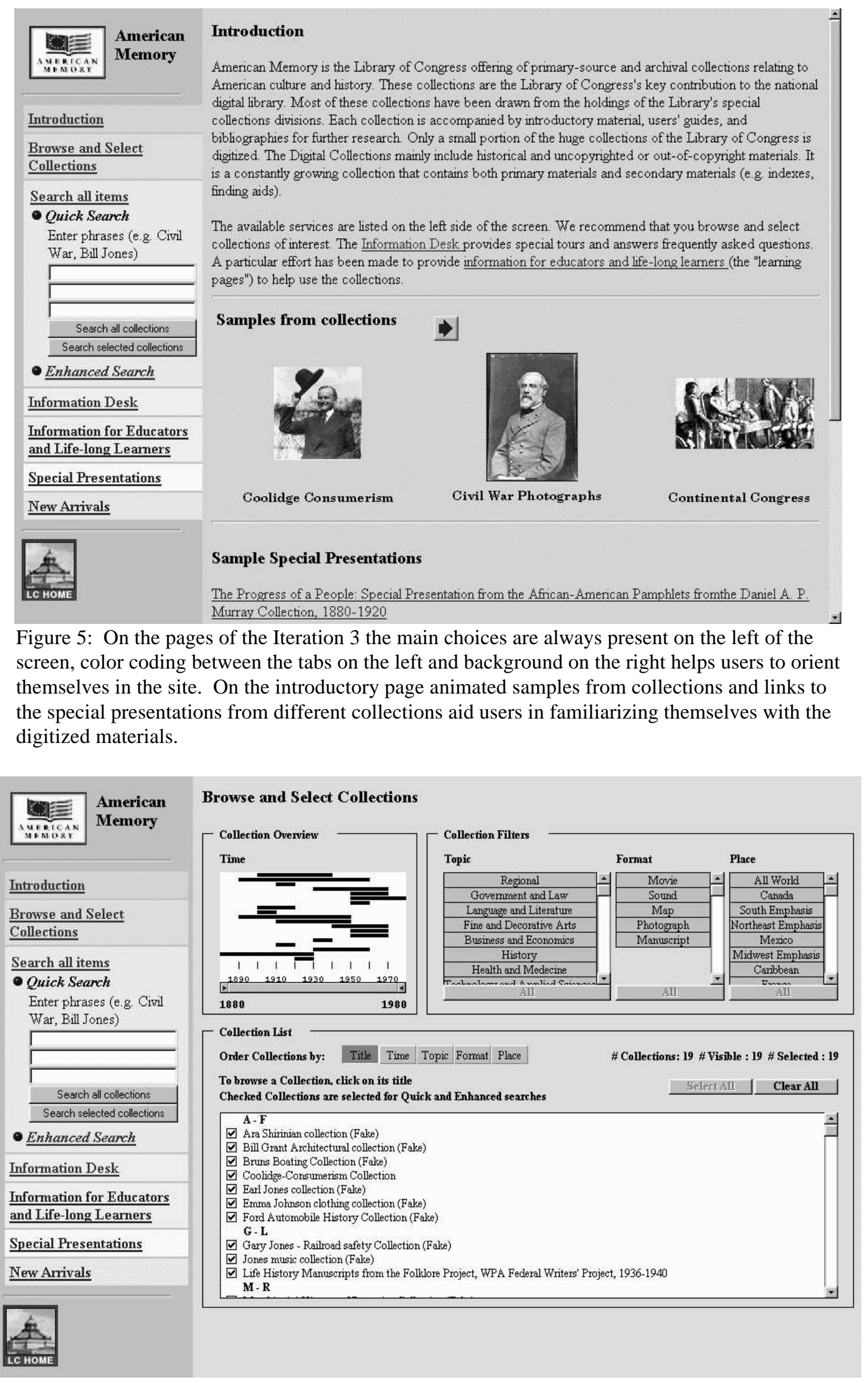

Figure 6: This figure shows the collection browser page with starfield display and closely coupled result lists. Filters here are represented by dynamic query interface widgets. 